\title{
Neural correlates of negative and disease-specific emotional stimuli in panic disorder: a functional magnetic resonance imaging study
}

\author{
Fabiana L. Lopes, ${ }^{1,2}$ iD Clara G.F. Faria, ${ }^{1}$ iD Gisele P. Dias, ${ }^{1,3}$ Mariana B. Mallmann, ${ }^{1}$ Victoria \\ Mendes, ${ }^{1}$ Natia Horato, ${ }^{1}$ Valfrido L. de-Melo-Neto, ${ }^{1}$ Andre B. Veras, ${ }^{1}$ Fabio V. Magalhães, ${ }^{4}$ Dolores \\ Malaspina, ${ }^{5}$ Antonio E. Nardi ${ }^{1}$ iD \\ ${ }^{1}$ Laboratório de Pânico e Respiração, Instituto de Psiquiatria, Universidade Federal do Rio de Janeiro, Rio de Janeiro, RJ, Brazil. ${ }^{2}$ Intramural \\ Program, National Institute of Mental Health (NIMH), National Institutes of Health (NIH), Bethesda, MD, USA. ${ }^{3}$ Institute of Psychiatry, \\ Psychology and Neuroscience, King's College London, London, United Kingdom. ${ }^{4}$ Clínica de Diagnóstico por Imagem, Rio de Janeiro, RJ, \\ Brazil. ${ }^{5}$ Icahn School of Medicine at Mount Sinai, New York, NY, USA.
}

\begin{abstract}
Objective: Decades of research have highlighted the involvement of the prefrontal cortex, anterior cingulated cortex, and limbic areas (amygdala) in panic disorder (PD). However, little attention has been given specifically to the inferior frontal gyrus. The current study aimed to investigate the neural substrates, including the inferior frontal gyrus, of both panic-related and negative conditions among individuals with PD and healthy controls.

Methods: We examined 13 medication-free PD patients and 14 healthy controls with functional magnetic resonance imaging (fMRI) during exposure to negative and neutral pictures and a set of specific panic-related pictures.

Results: Subtraction between the conditions indicated activation of the left amygdala region and the right inferior frontal gyrus in PD patients during the specific panic-related condition, whereas the left amygdalar region and left inferior frontal gyrus were activated during the negative condition in controls. Conclusion: These results suggest that in patients with PD, a prominent bottom-up process is involved in specific panic-related conditions, which might be associated with weak modulation of the left frontal area. These data add to our current understanding of the neural correlates of PD and can contribute to future clinical interventions targeting the functional reestablishment of these regions.
\end{abstract}

Keywords: Panic disorder; inferior frontal gyrus; amygdala; fMRI

\section{Introduction}

Panic disorder (PD) with or without agoraphobia is a potentially disabling condition with a lifetime prevalence of approximately 3 to $5 \% .^{1,2}$ Although efficacy studies suggest a good pattern of response to pharmacological and/or psychotherapeutic treatments, effectiveness and naturalistic studies have demonstrated that near $70 \%$ of treatment-seeking PD patients remain ill for longer periods and commonly relapse. ${ }^{3,4}$ Twin studies have shown a substantial heritable component in PD, ${ }^{5}$ but little is still known about the specific neurobiological mechanisms that mediate vulnerability to PD.

During the last decade, there has been a race to elucidate the biological bases of anxiety disorders through neuroimaging studies in order to develop more effective treatments. Advances in functional neuroimaging have helped clinical researchers make significant strides in this

Correspondence: Fabiana L. Lopes, 35 Convent Drive, 1A-207, Bethesda, MD, USA, 20850.

E-mail: lopes.fabiana@gmail.com

Submitted Oct 16 2020, accepted Jan 07 2021, Epub Mar 262021. field, allowing differentiation of neural responses between anxiety disorder patients and healthy controls. ${ }^{6}$ Compared to healthy individuals, the amygdala and insula (major regions involved in the fear network) are hyperactive in patients with anxiety disorders. ${ }^{6,7}$ However, despite a wealth of research on the neural correlates of anxiety disorders, surprisingly little is known about the neurobiology of PD. This can be attributed, in part, to the fact that "anxiety disorders" is a large umbrella term and that most studies focus on other anxiety disorders, such as post-traumatic stress disorder, generalized anxiety disorder, and social phobia rather than PD. ${ }^{8}$ Moreover, PD is a heterogeneous condition encompassing anticipatory anxiety, phobic avoidance, and panic attacks (PAs) - the core phenomena of the disorder. ${ }^{9}$ It has long been suggested that the first two components of PD are related to the fear reaction model, which is corroborated by consistent behavioral and physiological
How to cite this article: Lopes FL, Faria CGF, Dias GP, Mallmann $\mathrm{MB}$, Mendes V, Horato $\mathrm{N}$, et al. Neural correlates of negative and disease-specific emotional stimuli in panic disorder: a functional magnetic resonance imaging study. Braz J Psychiatry. 2021;43:605612. http://dx.doi.org/10.1590/1516-4446-2020-1573 
data. On the other hand, spontaneous and unexpected PAs, described as a core phenomenon of the disorder, have been associated with dysregulation of homeostatic function along the brainstem area.

The current theoretical model of fear neurocircuitry in PD proposes that the prefrontal cortex (PFC) fails in its modulatory role for the amygdala, leading to autonomic and behavioral activation. ${ }^{10}$ Preclinical models and neuroimaging studies with healthy controls have shown a direct connection between amygdalar and thalamic structures, showing an automatic response pattern to threats. A second, slower, amygdalar pathway involves superior cortical areas, comprising a negative feedback mechanism. ${ }^{10}$ Reappraisal and emotional evaluation studies of healthy subjects have shown that frontal activation is correlated with amygdala inhibition, which suggests that the former modulates the latter. ${ }^{11}$ It is plausible that different brain structures underpin unexpected PAs or the full-blown disorder. ${ }^{9}$

Current neuroimaging studies in PD are based on visual, ${ }^{12-17}$ auditory, ${ }^{18,19}$ or imagery exposure ${ }^{20}$ paradigms to general threat-related stimuli. In a spectroscopy study, Akiyoshi et al. ${ }^{16}$ found significantly lower left frontal oxyhemoglobin levels in PD patients than controls in visual anxiety-relevant or irrelevant situations, which suggests hypoactivity in the left frontal cortex. On the other hand, other researchers ${ }^{21}$ have reported a rightward shift in asymmetry within the posterior inferior frontal cortex. Nevertheless, several limitations have been highlighted in PD studies involving functional magnetic resonance imaging (fMRI), such as not including healthy volunteers or control groups with other anxiety disorders. ${ }^{22}$

To our knowledge, no studies have used fMRI and panic-relevant visual stimuli in a medication-naïve PD population and a healthy control group. We used a group of panic-specific anxiogenic pictures that had been validated in an independent PD sample at our center, which consisted of fearful and avoided daily situations, such as public transport, crowded places, and queues. ${ }^{23}$ The International Affective Picture System (IAPS), a wellvalidated collection of visual images was used for the negative and neutral pictures. ${ }^{24}$ Since inducing PAs through neuroimagery has a limited application due to general vasoconstriction properties, ${ }^{10}$ we decided to apply a paradigm with specific anxiogenic, but not panicogenic, stimuli in a PD sample compared to a healthy control group. Therefore, we used a baseline rather than a panicogenic paradigm, hoping to determine the neural substrates involved in the emotional and behavioral components of PD rather than actual PAs. The aim of the present study was to investigate the neural substrate of both panic-related and negative conditions among PD subjects and healthy controls by addressing the following questions: 1) Do PD subjects in panic-related conditions have the same response patterns as healthy subjects under a fearful (negative) condition?; and 2) Is the neural substrate of a negative condition a byproduct of emotional processing, reflecting the fear circuitry in healthy subjects? To answer these questions, we conducted an fMRI study with visual stimuli and hypothesized that each group would have different neural patterns when panic-related and neutral stimuli were contrasted in PD patients and negative vs. neutral stimuli in healthy subjects. Based on the prevailing theoretical models, ${ }^{8}$ we expected to find the same neural circuitries in PD patients and healthy subjects in the panic-related condition and negative situation, respectively.

\section{Methods}

\section{Subjects}

Thirteen PD patients with or without agoraphobia (seven men and six women, $35.3 \pm 9.1$ and $35.4 \pm 10.7$, mean age \pm standard deviation [SD] respectively) and fourteen healthy comparison subjects (nine men and five women, $28.7 \pm 3.7$ and $28.5 \pm 3.8$, mean age $\pm S D$, respectively) were enrolled in the study. The PD patients were selected during their first appointment at the Laboratório de Pânico e Respiração, Universidade Federal do Rio de Janeiro, after reporting recurrent PAs in the 4 weeks prior to the study. To be included, the patients had to be between 18 and 65 years of age and in good global clinical condition. The exclusion criteria for all participants were: pregnancy, a metallic prosthesis and/or pacemaker, and significant cardiovascular, respiratory, or neurological abnormalities. The patients with PD had to be free of any psychoactive medications in the past 4 weeks and have none of the following comorbidities: schizophrenia, bipolar disorder, obsessive-compulsive disorder, social phobia, current major depressive disorder, eating disorder, and posttraumatic stress disorder. Substance abuse or dependence in the 6 months prior to the study was another exclusion criterion. The participants were interviewed and diagnosed using the Mini International Neuropsychiatric Interview. Additional instruments used to assess PD severity included the Hamilton Anxiety Rating Scale, the Sheehan Panic Disorder Scale, and the Visual Analogue Scale for Anxiety.

The healthy controls were free of lifetime psychiatric disorders according to the DSM-IV-TR.

\section{Activation stimuli}

The emotional visual stimuli consisted of 96 pictures in a blocked presentation (32 panic-related, 32 negative, and 32 neutral). Each block consisted of 16 pictures and each of the three categories was presented twice using different pictures. The negative block consisted of disturbing mutilation pictures selected from the IAPS. This dataset of pictures, designed for studying emotion and attention, has been used in an array of psychophysiological studies and includes 956 color images ranging from everyday objects and scenes to extremely horrifying pictures - such as mutilations. ${ }^{24}$ Objects and utensils, also drawn from this catalog, were used in the neutral category. The panic-related pictures consisted of daily distressing situations for patients with PD (e.g., queues, crowded and open places, bridges, underwater scenes, public transportation) and had been previously evaluated in another group of patients with PD from our center $(n=10)$. These pictures did not differ in valence 
$(5.09 \pm 1.09$ vs. $4.59 \pm 2.73$, mean \pm SD for specific panic related vs. mutilation pictures respectively, $p=0.32$ ) or arousal $(5.09 \pm 0.85$ vs. $5.64 \pm 1.53$, mean \pm SD for panicrelated vs. mutilation pictures respectively, $p=0.06$ ) from the IAPS mutilation pictures, but were significantly more anxiogenic according to the Subjective Units of Distress Scale (4.54 \pm 1.23 vs. $3.18 \pm 2.32$, mean \pm SD for anxiogenic vs. mutilation respectively, two-tailed $t$-test, $\mathrm{p}<0.005)$. Each picture was shown for 3 seconds and each block took 48 seconds. Each block was preceded and followed by a grey screen that lasted for 24 seconds. The duration of the whole experiment was 7.6 minutes. The participants were instructed to simply passively view the picture without trying to modulate any associated feelings.

\section{FMRI data acquisition}

Magnetic resonance images were acquired with a $1.5 \mathrm{~T}$ Siemens Magneton Avanto whole-body scanner equipped with echo-planar imaging using a standard head coil for radio-frequency transmission and signal reception. $A$ 3D T1-weighted structural image was acquired for each participant as an anatomical reference. Using a sagittal scout image, a total of 144 contiguous slices were acquired (1.1 mm thickness) with a repetition time (TR) of $1,900 \mathrm{~ms}$, echo time (TE) of $3.93 \mathrm{~ms}$, and interval time (TI) of 1,100 ms. A flair in the axial plane was performed with 20 slices (5 mm thickness, TR $=9,650 \mathrm{~ms}$, TE $=87 \mathrm{~ms}$, $\mathrm{TI}=2,500$ $\mathrm{ms})$. Single shot T2 echo-planar imaging for blood oxygen level-dependent (BOLD) functional brain imaging covered the whole brain. Twenty-four slices were acquired with 140 repetitions and a matrix of $64 \times 64$ (3 mm thickness, field of view $=192 \mathrm{~mm}, \mathrm{TR}=3,000 \mathrm{~ms}, \mathrm{TE}=46 \mathrm{~ms}, \mathrm{TI}=0.75 \mathrm{~ms}$ ).

\section{Image processing and analysis}

Image processing and statistical analysis were performed using Statistical Parametric Mapping (SPM5; www.fil.ion. ucl.ac.uk). Standard pre-processing consisted of slicetime correction, realignment (motion correction), and masked normalization of each participant's echo planar imaging data to the Montreal Neurological Institute/International Consortium of Brain Mapping template. Images were resampled into this space with $2-\mathrm{mm}$ isotropic voxels and smoothed using a Gaussian kernel with fullwidth half maximum of $8 \mathrm{~mm}$.
On the single subject level, the BOLD response was analyzed in the context of a general linear model. The signal time course for each subject was modeled with a boxcar function and trials were convolved with the canonical hemodynamic response function to form regressors. We applied three regressors of interest. Our experimental conditions were panic-related, negative, and neutral pictures. A random effects analysis was used to analyze data at a group level. Statistical parametric maps were implemented. Each condition was contrasted with the control condition (grey screen), creating one contrast image per subject for each condition. These images were entered into a one-sample $t$-test to investigate significant activation during each task. Significant signal changes for each contrast were assessed using $t$-statistics on a voxelby-voxel basis. Neutral images were subtracted from the panic-related and negative conditions to determine the neural substrates of emotional picture processing. Panicrelated images were also subtracted from the negative condition. A statistical threshold of $p<0.001$ was used to identify significant voxels for all comparisons. For all analyses, the region names, z-values, and coordinates of activated foci are listed in the tables.

\section{Ethics statement}

The participants provided written informed consent after receiving a full explanation of the study's procedures.

\section{Results}

The sample's clinical and demographic characteristics are shown in Table 1. One male subject with PD was excluded due to a temporal arachnoid cyst. The participants had a mean (SD) score of 24.79 (9.17) on the Hamilton Anxiety Rating Scale, 52.0 (24.86) on Sheehan PD scale, and 6.07 (2.40) on the Visual Analogue Scale for Anxiety. These findings are consistent with moderate severity. No significant differences were observed between PD with or without agoraphobia (Table 1).

The functional imaging data (Table 2 and Figures 1, 2 and 3 ) illustrate condition effects within groups.

In the PD group, subtracting the neutral condition from the panic-related condition (panic-related $>$ neutral) revealed that the predominant neural substrates involved when processing panic panic-related pictures are in the

Table 1 Clinical and demographic characteristics of the sample

\begin{tabular}{|c|c|c|c|c|c|c|}
\hline & PD with agoraphobia $(n=7)$ & PD without agoraphobia $(n=5)$ & PD total $(n=12)$ & $\mathrm{F}$ & df & $p$-value \\
\hline \multicolumn{7}{|c|}{ Sex*, n (\%) } \\
\hline Female & $5(71.42)$ & $1(20.00)$ & $6(50.00)$ & & & \\
\hline Male & $2(28.57)$ & $4(80.00)$ & $6(50.00)$ & & & \\
\hline & & & & 0.41 & 10 & 0.92 \\
\hline Age* & $33.86(6.49)$ & $35.60(12.81)$ & $34.58(9.14)$ & 1.99 & 10 & 0.76 \\
\hline HAM-A & $27.67(7.43)$ & $19.60(10.52)$ & $24.79(9.17)$ & 0.29 & 12 & 0.12 \\
\hline SPS & 56.89 (20.04) & $43.20(32.47)$ & $52.00(24.86)$ & 4.35 & 12 & 0.34 \\
\hline VAS-A & $6.44(2.65)$ & $5.40(1.94)$ & $6.07(2.40)$ & 0.94 & 12 & 0.46 \\
\hline
\end{tabular}

Data presented as mean (standard deviation), unless otherwise specified.

$\mathrm{df}=$ degrees of freedom; HAM-A = Hamilton Anxiety Rating Scale; PD = panic disorder; SPS = Sheehan Panic Disorder Scale; VAS-A = Visual Analogue Scale for Anxiety.

* Data available for only 12 PD subjects with or without agoraphobia. 
Table 2 Significant BOLD fMRI responses for the subtraction effect comparisons

\begin{tabular}{|c|c|c|}
\hline Main effects & MNI coordinates $(x, y, z)$ & Cluster size (number of voxels) \\
\hline \multicolumn{3}{|l|}{ PD patients } \\
\hline \multicolumn{3}{|l|}{ Panic $>$ Neutral } \\
\hline Left periamygdalar cortex & $-39,-6,-13$ & 49 \\
\hline Right IFG & $51,-6,9$ & 66 \\
\hline \multicolumn{3}{|l|}{ Negative $>$ Neutral } \\
\hline Left PFC & $-26,53,5$ & 223 \\
\hline Right IFG & $39,1,27$ & 93 \\
\hline Left parietal cortex & $-56,-46,40$ & 57 \\
\hline \multicolumn{3}{|l|}{ Panic $>$ Negative } \\
\hline Left periamygdalar cortex & $-36,0,-12$ & 49 \\
\hline Right IFG & $57,-3,24$ & 83 \\
\hline \multicolumn{3}{|l|}{ Negative $>$ Panic } \\
\hline Left PFC & $-22,55,0$ & 125 \\
\hline \multicolumn{3}{|l|}{ Control subjects } \\
\hline \multicolumn{3}{|l|}{ Panic $>$ Neutral } \\
\hline Insular cortex & $-31,20,-7$ & 13 \\
\hline \multicolumn{3}{|l|}{ Negative $>$ Neutral } \\
\hline Left periamygdalar cortex & $-27,-5,-5$ & 47 \\
\hline Left IFG & $-54,1,21$ & 85 \\
\hline \multicolumn{3}{|l|}{ Panic > Negative } \\
\hline Artifacts & - & - \\
\hline \multicolumn{3}{|l|}{ Negative $>$ Panic } \\
\hline Left IFG & $-53,3,24$ & 68 \\
\hline Left PFC & $-7,47,-8$ & 73 \\
\hline
\end{tabular}

BOLD = blood oxygen level dependence; fMRI = functional magnetic resonance imaging; IFG = inferior frontal gyrus; MNI = Montreal Neurological Institute; PD = panic disorder; PFC = prefrontal cortex.
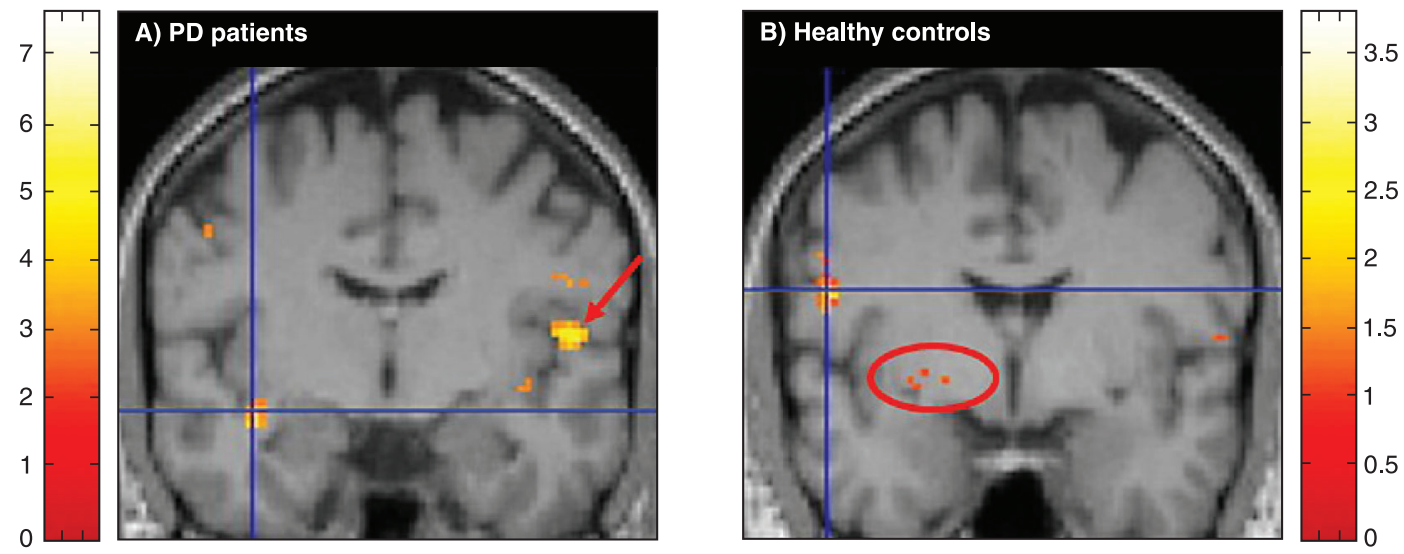

Figure 1 A) Hemodynamic response in PD patients during anxiogenic > neutral, showing activation in the left periamygdalar cortex and right IFG (red arrow [max $t$-value $=2.96]$ ). B) Healthy controls during negative $>$ neutral with activation in left periamygdalar cortex (red circle) and left IFG (max $t$-value =1.18). IFG = inferior frontal gyrus; PD = panic disorder.

left periamygdalar cortex $(-39,-6,-13, \mathrm{~T}=2.96)$ and the right inferior frontal gyrus (IFG)(51, -6, 9, $\mathrm{T}=2.96)$ (Figure $1 \mathrm{~A})$. There was no significant activated area in healthy controls and their results were considered artefacts.

For the PD group, the negative vs. neutral (negative $>$ neutral) contrast yielded a significant effect in the left PFC $(-26,53,5, T=5.45)$, the right IFG $(39,1,27$, $\mathrm{T}=5.45)$, and the left parietal cortex $(-56,-46,40, \mathrm{~T}=$
5.45) (Figure 2). In contrast, in healthy controls there was activation in the left periamygdalar cortex $(-27,-5$, $-5, \mathrm{~T}=1.18)$ and the left IFG $(-54,1,21, \mathrm{~T}=1.18)$ (Figure 1B).

Panic-related vs. negative contrast in the PD patients indicated increased activation in the left periamygdalar cortex $(-36,0,-12, \mathrm{~T}=2.96)$ and the right IFG $(57,-3,24$, $\mathrm{T}=2.96)$. Finally, negative vs. panic-related contrast showed activation of the left PFC $(-22,55,0, T=5.45)$ in 

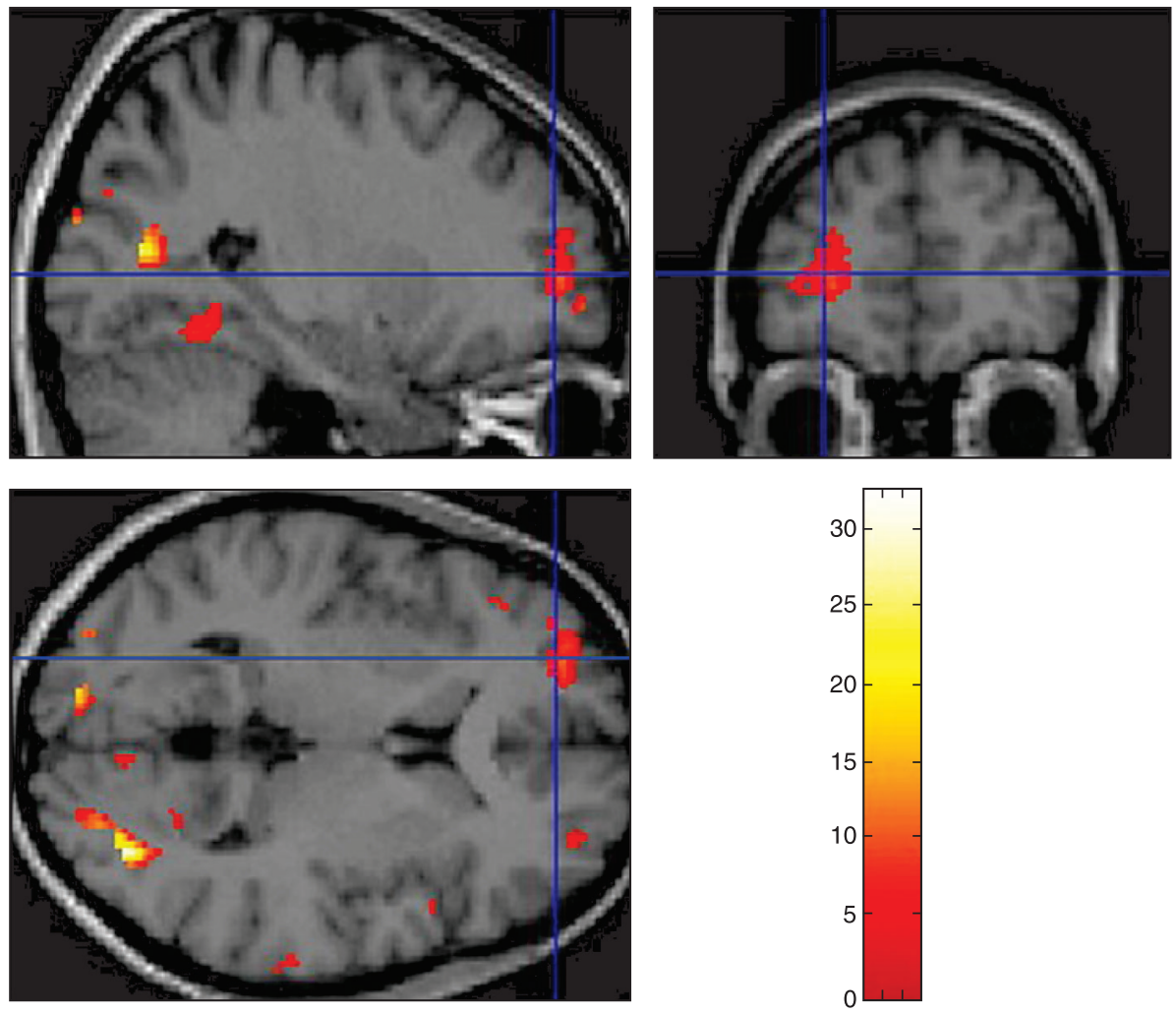

Figure 2 Blood oxygen level dependence response in panic disorder patients during the negative-minus-neutral condition. The left prefrontal cortex was recruited in panic disorder patients during the subtraction of negative and neutral conditions. Max t-value $=5.45$.

the PD group, whereas there was activation of the left IFG in the control group $(-53,3,24, T=1.18)$ and left PFC $(-7$, $47,-8, T=1.18$ ) (Table 2 and Figure 3 ).

\section{Discussion}

In the present study, the passive viewing of panic-related/ agoraphobic, negative, and neutral pictures was used to investigate the neuroanatomical circuitry of PD patients and healthy controls. The main regions recruited in both the PD and control groups during the experiment were the IFG, amygdala, and insula. Thus, this study clearly demonstrated an underlying neurocircuitry that encompasses, at least in part, the theoretical model of the fear circuitry. ${ }^{10}$

As expected, different neural patterns were observed in each group when contrasting panic-related with neutral stimuli. Interestingly, the pattern of response in the PD group during the panic-related condition overlapped with that of the control group during the negative condition, differing only in lateralization of the IFG. While the left IFG was activated in the control group during the negative block, the right IFG in the disease-specific condition was activated in the PD group. Such findings corroborate previous data that the left IFG is activated in healthy subjects in several visual stimulus paradigms and/or a classic or emotional Stroop task. ${ }^{25-28}$ Lidaka et al. ${ }^{28}$ correlated structures in the amygdala and cortex in healthy subjects while they determined the sex of faces with negative, positive, or neutral emotion. They found that left amygdala activity was positively correlated with left PFC activity in the negative-minus-neutral condition. Thus, the left amygdala and left IFG activation we found in healthy controls may corroborate Lidaka et al.'s hypothesis that the processing of negative expressions is modulated by the neural interaction between the PFC and amygdala in the left hemisphere.

On the other hand, the right hemisphere has been implicated in some studies of patients with PD. ${ }^{15,16,29,30}$ It is beyond the scope of this article to analyze brain asymmetry. Furthermore, emotional lateralization is complex and may depend on various factors, ${ }^{29}$ and studies concerning the PFC are still discordant. ${ }^{22,31}$ However, certain points should be considered. The right hemisphere is involved in vigilance and autonomic arousal. ${ }^{27,32}$ In particular, the right anterior insular/opercular cortex have been implicated in increased interoceptive attention and representation of visceral responses accessible to awareness, thus providing a substrate for subjective feeling states. ${ }^{33}$ In addition, the right IFG is involved in detecting salient stimuli that are behaviorally relevant. ${ }^{34}$ Thus, it is possible that our PD group activated mnemonic resources while visualizing panicrelated situations, which triggered a state of hyperarousal in which they were "concerned" about the somatic symptoms normally present during PAs. Alternatively, such activation could lead to an anticipatory anxious state. $^{15}$ 

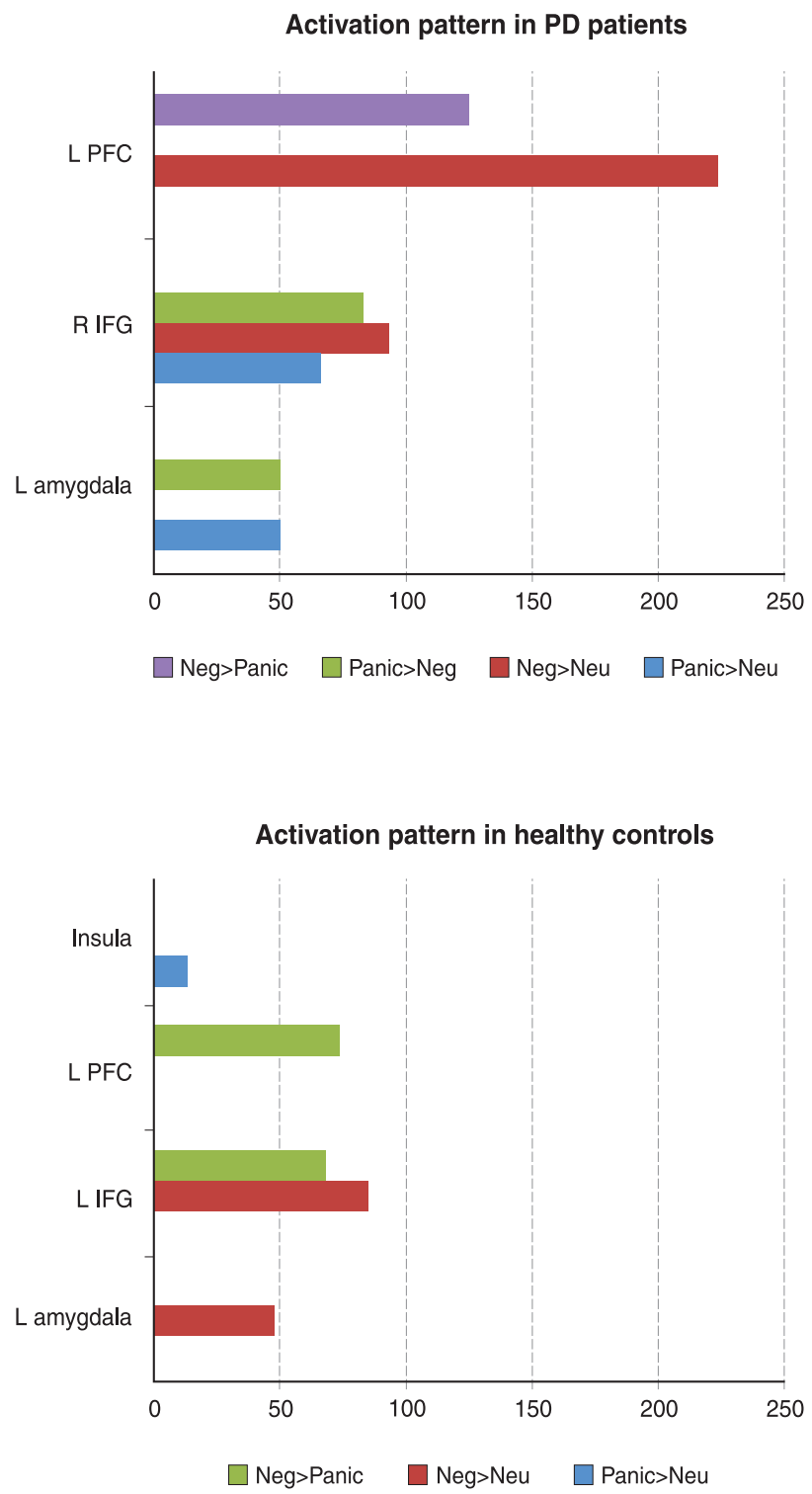

Figure 3 Interaction between activated areas and their respective peak voxel values in PD patients and healthy controls. The $x$ coordinate represents peak voxel values, which correspond to activation during one of the three conditions. IFG = inferior frontal gyrus; $L=$ left; Neg = negative; $\mathrm{Neu}=$ neutral; $\mathrm{PD}=$ panic disorder; $\mathrm{PFC}=$ prefrontal cortex; $\mathrm{R}=$ right.

Attentional bias has been consistently reported in $\mathrm{PD},{ }^{13,34}$ including descriptions of extensive mnemonic processing of threat-related stimuli as a characteristic of PD. ${ }^{19,34}$ These findings could suggest that panic is activated by a top-down mechanism arising from the central selection of pathological response patterns due to conditioning by previous experience or to the interaction between predisposition and experience. ${ }^{35}$ However, our data suggest that the neuronal substrate of emotional processing seems to be different for general threat cues and disease-specific cues in PD patients, given that the engaged neurocircuitry is more widespread during negative viewing, including frontoparietal activation without the involvement of subcortical limbic structures (i.e., the amygdala). Thus, we raise the hypothesis that during the negative condition in PD (and in threatening stimuli in general) a higher cognitive top-down mechanism predominates, whereas in the specific panic condition (with diseasespecific amygdala activity) an emotional-generative mechanism is initiated. ${ }^{6}$ At this point, the right inferior frontal cortex may be responding with a cognitive elaboration, ${ }^{13}$ thus reflecting attentional switching. Interestingly, a recent meta-analysis of 367 task-related fMRI experiments in mood disorders, posttraumatic stress disorder, and anxiety disorders, comprising data from 4,507 patients and 4,755 controls, found that the right inferior PFC is critically involved in inhibiting contextually inappropriate cognitive, affective, and motor responses. Furthermore, the authors detected statistically robust transdiagnostic clusters of hypoactivation in the inferior PFC/insula, the inferior parietal lobule, and the putamen in the patient group. ${ }^{8}$ Thus, we infer that the right IFG activation of PD subjects in the disease-specific condition represents a general disruption in salience processing and inhibitory control rather than right-sided fronto-parietal hyperactivity, as was previously thought.

Lesion studies show that stopping an initiated response depends on the integrity of the right IFG and the presupplementary motor area. ${ }^{34,36}$ Additionally, diffusion tensor imaging tractography shows that the IFG and pre-supplementary motor area are structurally connected to one another and the basal ganglia, comprising a possible network for action control. ${ }^{37}$ Notably, recent fMRI studies show that the right IFG is active when preparing to stop, as well as during outright stoppage. ${ }^{37}$ Alternatively, PD patients may activate this region during the fightor-flight response to a threatening cue, as an output response of the fear cascade. In fact, there is open debate about which brain areas are involved in PD: limbic structures may be more relevant for anticipatory anxiety/ phobic avoidance, while other structures, such as the brainstem, may be more "crucial" for PAs. ${ }^{9}$ It should be pointed out that our study did not involve a panicogenic paradigm and the participants did not have PAs during the experiment. In addition, our sample had moderate to severe levels of anxiety according to the baseline instruments. Therefore, our findings might reflect anticipatory anxiety or the consequences of intermittent PAs. It seems less likely they represent phobic anxiety, since there was no difference between PD with and without agoraphobia. Moreover, the participants enrolled in this study were in the acute phase, since it was their first appointment and they were medication-naïve.

It is well known that PD patients have difficulty identifying and managing a range of emotional experiences, as well as a tendency to interpret ambiguous internal and external stimuli as threatening. ${ }^{38}$ The high prevalence of alexithymia found in PD patients ${ }^{38}$ may be linked with a bias toward somatic concerns in stressful situations and difficulty establishing a relationship between PA and emotional triggers, ${ }^{38}$ as well as the high prevalence of alexithymia in drug-free PD subjects and impairment in verbal cognitive abilities, which suggests a poor 
symbolization processes in these patients. On the other hand, the literature demonstrates that successful cognitive behavior therapy (CBT) is associated with higher recruitment of the left IFG. The left IFG is involved primarily in cognitive function (e.g., attention, execution control, reasoning, verbalization) and has been consistently linked to language processing and maintaining verbal information. ${ }^{23,24,39}$ It could be argued that CBT acts by recruiting the left IFG, which is hyporeactive in PD patients, thus attenuating symptoms and leading to activation of top-down inhibitory mechanisms.

Regarding the right IFG and amygdala activation pattern, our data is in line with other studies. ${ }^{11,13,14}$ Wittman et al. ${ }^{15}$ used a set of agoraphobic pictures, attributing the results to anticipatory anxiety. However, they did not use a control group. In a case-control study with $\mathrm{fMRI}$, Van den Heuvel ${ }^{13}$ investigated an emotional Stroop task in PD patients. The patients displayed increased activity in the right amygdala and hippocampus in response to panic-related vs. neutral words; additional activation - which was more pronounced in the right hemisphere - was found in PFC areas (medial, ventrolateral, and dorsolateral), the anterior cingulate cortex, the middle temporal gyrus, and the inferior parietal lobe. In the controls, panic-related words did not elicit substantial activation. Their findings agree with our data in that the widespread activation of prefrontal and temporal areas during threat stimuli suggests a more generalized attentional bias to threat cues in PD. Interestingly, amygdala activation did not occur during this condition, which indicates that it is disease-specific (in our results, the amygdala was not activated during the negative condition). Finally, similar to our findings, they observed increased recruitment of the right dorsolateral PFC during the panic-related condition, which they attributed to increased cognitive elaboration.

On the other hand, other studies have found increased recruitment of the left IFG. ${ }^{39,40}$ Dressler et al. ${ }^{36}$ conducted an $\mathrm{fMRI}$ study with an emotional Stroop task in PD patients and healthy controls. It is important to point out that they used a heterogeneous sample of participants who had received or were receiving psychotherapy and/or pharmacological treatment at the time of the study. Kircher et al. ${ }^{39}$ showed that after CBT, PD patients had lower left IFG activation for the conditioned response than the control group. The authors linked these findings to cognitive processes (in the IFG) that could more easily trigger emotional responses related to fear network activity in PD patients than controls. However, Shurick \& Gross ${ }^{8}$ made three important points about the data of Kircher et al. ${ }^{39}$ that suggest caution. First, it is not clear whether the IFG data are best interpreted as initial hyperactivity that is normalized by CBT or as a sign of exaggerated attempts to engage the IFG to regulate emotions at T1 (baseline). Second, the lack of a group-bytime interaction limits the robustness of the findings. Third, none of the differences between the PD and control groups were evident during late acquisition or extinction. Moreover, other studies have demonstrated bilateral engagement of the dorsolateral PFC during spontaneous $\mathrm{PA}$ and after effective treatment with paroxetine. ${ }^{41}$
In summary, we found two distinct neural substrates of visual processing in PD: more widespread activation (fronto-parietal) for general threatening cues (negative stimuli); and right dorsolateral PFC (IFG) and left amygdalar activation for panic-related cues. It is noteworthy that the amygdala was activated only during the specific panic stimuli, which supports a bottom-up regulation model. We hypothesize that the IFG functions as a regulatory region. Left IFG hyporegulation of the amygdala could be one mechanism involved in the neurobiology of PD. We cannot conclude from our data whether the right IFG recruitment in PD represents hyperreactivity or is a sign of an exaggerated attempt to engage this region to regulate the automatic response evoked by the amygdala. We addressed three considerations about right IFG activity. First, it could represent a cognitive elaboration. ${ }^{15}$ Second, it belongs to the interoceptive network and could reflect a bias towards somatic concerns in threatening situations. Finally, it could represent a state of "preparing for action." If such were the case, it could also indicate that a fight-freezeflight response underlies the prevailing model of fear circuitry in PD. We speculate that improvement due to CBT, which is consistently demonstrated in the literature, could be the result of engaging the left IFG.

This study, however, is limited by its small sample size and a lack of cognitive control during the experiment. However, we specifically instructed the participants regarding passive viewing, which is consistent with the standard protocol model. From a clinical point of view, it would be interesting to apply this paradigm in a sample of patients with PD and other anxiety disorders after treatment in two arms: pharmacological and psychotherapeutic. Future studies should also correlate the identified neural substrates with clinical parameters and perform intergroup analyses. Moreover, future investigations could benefit from a functional connectivity approach, thus indicating not only which brain regions are activated during disease-specific conditions, but their patterns of interactivity. Since we did not perform a functional connectivity analysis, we cannot state that our findings reflect right IFG hyperactivation or a lack of left IFG modulation. We observed a BOLD signal in the right IFG that could also represent heterogeneity. Our results should be considered preliminary until performed in a larger sample with connectivity models, which would provide a more precise understanding of the functional network underlying PD.

Finally, although obtained through a simple subtractive approach, our data can still provide novel and relevant information about which regions are activated by a particular situation in patients with PD and, importantly, about the boundary conditions for involvement of a particular brain region (IFG/amygdala), with consequent insight into the functional neuroanatomy of PD and human emotion. $^{31}$

\section{Acknowledgements}

This study has been funded by grants from the Coordenação de Aperfeiçoamento de Pessoal de Nível Superior (CAPES; 0111-05-8), Conselho Nacional de 
Desenvolvimento Científico e Tecnológico (CNPq), Fundação de Amparo à Pesquisa do Estado do Rio de Janeiro/Programa de Apoio a Núcleos de Excelência (FAPERJ/PRONEX), and Instituto Nacional de Ciência e Tecnologia - Medicina Translacional (INCT-TM)/CNPq.

\section{Disclosure}

The authors report no conflicts of interest.

\section{References}

1 Grant BF, Hasin DS, Stinson FS, Dawson DA, Goldstein RB, Smith S, et al. The epidemiology of DSM-IV panic disorder and agoraphobia in the United States: results from the National Epidemiologic Survey on Alcohol and Related Conditions. J Clin Psychiatry. 2006;67:363-74.

2 Wittchen HU, Jacobi F. Size and burden of mental disorders in Europe - a critical review and appraisal of 27 studies. Eur Neuropsychopharmacol. 2005; 15:357-76.

3 Bruce Se, Yonkers KA, Otto MW, Eisen JL, Weisberg RB, Pagano M, et al. Influence of psychiatric comorbidity on recovery and recurrence in generalized anxiety disorder, social phobia, and panic disorder: a 12-year prospective study. Am J Psychiatry. 2005;162:1179-87.

4 Roy-Byrne PP, Craske MG, Stein MB, Sullivan G, Bystritsky A, Katon $W$, et al. A randomized effectiveness trial of cognitive behavioral therapy and medication for primary care panic disorder. Arch Gen Psychiatry. 2005;62:290-8.

5 Kendler KS, Gardner CO, Prescott CA. Panic syndromes in a population-based sample of male and female twins. Psychol Med. 2001;31:989-1000.

6 Shurick AA, Gross JJ. Emotional reactivity and regulation in panic disorder: insights from a functional magnetic resonance imaging study of cognitive behavioral therapy. Biol Psychiatry. 2013;73:5-6.

7 Etkin A, Wager TD. Functional neuroimaging of anxiety: a metaanalysis of emotional processing in PTSD, social anxiety disorder, and specific phobia. Am J Psychiatry. 2007;164:1476-88.

8 Janiri D, Moser DA, Doucet GE, Luber MJ, Rasgon A, Lee WH, et al. Shared neural phenotypes for mood and anxiety disorders: a metaanalysis of 226 task-related functional imaging studies. JAMA Psychiatry. 2020;77:172-9.

9 Perna G, Guerriero G, Brambilla P, Caldirola D. Panic and the brainstem: clues from neuroimaging studies. CNS Neurol Disord Drug Targets. 2014;13:1049-56.

10 Gorman JM, Kent JM, Sullivan GM, Coplan JD. Neuroanatomical hypothesis of panic disorder, revised. Am J Psychiatry. 2000;157: 493-505.

11 Hariri AR, Mattay VS, Tessitore A, Fera F, Weinberg DR. Neocortical modulation of the amygdala response to fearful stimuli. Biol Psychiatry. 2003:53:494-501.

12 Pillay SS, Rogowska J, Gruber SA, Simpson N, Yurgelun-Todd DA. Recognition of happy facial affect in panic disorder: an fMRI study. J Anxiety Disord. 2007;21:381-93.

13 Van den Heuvel OA, Veltman DJ, Groenewegen HJ, Witter MP Merkelbach J, Cath DC, et al. Disorder-specific neuroanatomical correlates of attentional bias in obsessive-compulsive disorder, panic disorder, and hypochondriasis. Arch Gen Psychiatry. 2005;62:922-33.

14 Chechko N, Wehrle R, Erhardt A, Holsboer F, Czisch M, Samann PG. Unstable prefrontal response to emotional conflict and activation of lower limbic structures in remitted panic disorder. PLoS One. 2009;4: e5537.

15 Wittmann A, Schlagenhauf F, John T, Guhn A, Rehbein H, Siegmund $A$, et al. A new paradigm (Westphal-Paraadigm) to study the neural correlates of panic disorder with agoraphobia. Eur Arch Psychiatry Clin Neurosci. 2011;261:185-94.

16 Akiyoshi J, Hieda K, Aoki Y, Nagayama H. Frontal brain hypoactivity as a biological substrate of anxiety in patients with panic disorder. Neuropsychobiology. 2003;47:165-70.

17 Pillay SS, Gruber SA, Rogowska J, Simpson N, Yurgelun-Todd DA. fMRI of fearful facial affect recognition in panic disorder: the cingulate gyrus-amygdala connection. J Affect Disord. 2006;94:173-81.
18 Pfleiderer B, Zinkirciran S, Arolt V, Heindel W, Deckert J, Domschke $\mathrm{K}$. $\mathrm{fMRI}$ amygdala activation during a spontaneous panic attack in a patient with panic disorder. World J Biol Psychiatry. 2007;8:269-72.

19 Maddock RJ, Buonocore MH, Kile SJ, Garrett AS. Brain regions showing increased activation by threat-related words in panic disorder. Neuroreport. 2003;14:325-8.

20 Bystritsky A, Pontillo D, Powers M, Sabb FW, Craske MG, Bookheimer SY. Functional MRI changes during panic anticipation and imagery exposure. Neuroreport. 2001;12:3953-7.

21 Nordahl TE, Stein MB, Benkelfat $C$, Semple WE, Andreason $P$, Zametkin A, et al. Regional cerebral metabolic asymmetries replicated in an independent group of patients with panic disorders. Biol Psychiatry. 1998;44:998-1006.

22 de Carvalho MR, Dias GP, Cosci F, de-Melo-Neto VL, Bevilaqua MC, Gardino PF, et al. Current findings off MRI in panic disorder: contributions for the fear neurocircuitry and CBT effects. Expert Rev Neurother. 2010;10:291-303.

23 First MB, Spitzer RL, Gibbon M, Williams JB. Structured Clinical Interview Diagnostic (SCID) for DSM-IV axis I Disorders - Clinician Version (SCID-CV). Washington: American Psychiatric Press; 1997.

24 Lang PJ, Bradley MM, Cuthbert BN. The international affective pictures system (IAPS). Technical manual and affective ratings. Gainsville: University of Florida; 1999.

25 Mincic AM. Neural substrates of the cognitive and emotional interference processing in healthy adolescents. Acta Neurobiol Exp (Wars). 2010;70:406-22.

26 Engels AS, Heller W, Mohanty A, Herrington JD, Banich MT, Webb $A G$, et al. Specificity of regional brain activity in anxiety types during emotion processing. Psychophysiology. 2007;44:352-63.

27 Compton RJ, Banich MT, Mohanty A, Milham MP, Herrington J, Miller $\mathrm{GA}$, et al. Paying attention to emotion: an $\mathrm{FMRI}$ investigation of cognitive and emotional stroop tasks. Cogn Affect Behav Neurosci. 2003;3:81-96.

28 lidaka T, Omori M, Murata T, Kosaka H, Yonekura Y, Okada T, et al. Neural interaction of the amygdala with the prefrontal and temporal cortices in the processing of facial expressions as revealed by fMRI. J Cogn Neurosci. 2001;13:1035-47.

29 Rauch SL, Shin LM, Wright Cl. Neuroimaging studies of amygdala function in anxiety disorders. Ann N Y Acad Sci. 2003;985:389-410.

30 Reiman EM, Raichle ME, Butler FK, Herscovitch P, Robins E. A focal brain abnormality in panic disorder, a severe form of anxiety. Nature. 1984;310:683-5.

31 Wager TD, Phan KL, Liberzon I, Taylor SF. Valence, gender, and lateralization of functional brain anatomy in emotion: a meta-analysis of findings from neuroimaging. Neuroimage. 2003;19:513-31.

32 Heller W, Nitschke JB, Lindsay DL. Neuropsychological correlates of arousal in self-reported emotion. Cogn Emot. 1997;11:383-402.

33 Critchley HD, Wiens S, Rotshtein P, Ohman A, Dolan RJ. Neural systems supporting interoceptive awareness. Nat Neurosci. 2004;7: 189-95.

34 Corbetta M, Shulman GL. Control of goal-directed and stimulusdriven attention in the brain. Nat Rev Neurosci. 2002;3:201-15.

35 Miller GA, Galanter E, Pribram KH. Plans and the structure of behavior. New York: Henry Holt; 1960.

36 Dressler T, Attar $\mathrm{CH}$, Spitzer C, Lowe B, Deckert J, Buchel C, et al. Neural correlates of the emotional stroop task in panic disorder patients: an event-related fMRI study. J Psychiatr Res. 2012;46:1627-34.

37 Swann NC, Cai W, Conner CR, Pieters TA, Claffey MP, George JS, et al. Roles for the pre-supplementary motor area and the right inferior frontal gyrus in stopping action: electrophysiological responses and functional and structural connectivity. Neuroimage. 2012;59:2860-70.

38 Galderisi S, Mancuso F, Mucci A, Garramone S, Zamboli R, Maj M. Alexithymia and cognitive dysfunctions in patients with panic disorder. Psychother Psychosom. 2008;77:182-8.

39 Kircher T, Arolt V, Jansen A, Pyka M, Reinhardt I, Kellermann T, et al. Effect of cognitive-behavioral therapy on neural correlates of fear conditioning in panic disorder. Biol Psychiatry. 2013;73:93-101.

40 Dresler T, Hahn T, Plichta MM, Ernst LH, Tupak SV, Ehlis AC, et al. Neural correlates of spontaneous panic attacks. J Neural Transm (Vienna). 2011;118:263-9.

41 Sim HB, Kang EH, Yu BH. Changes in cerebral cortex and limbic brain functions after short-term paroxetine treatment in panic disorder: an [F]FDG-PET pilot study. Psychiatry Investig. 2010;7: 215-9. 